Published in "Neuroscience Letters 548: 38-43, 2013"

which should be cited to refer to this work.

\title{
Impulsivity and home-cage activity are decreased by lentivirus-mediated silencing of serotonin transporter in the rat hippocampus
}

\author{
Francesca Zoratto ${ }^{\mathrm{a}, \mathrm{e}}$, Amanda L. Tringle $^{\mathrm{a}}$, Giancarlo Bellenchi ${ }^{\mathrm{b}}$, Luisa Speranza ${ }^{\mathrm{b}, \mathrm{c}}$, \\ Domenica Travaglini $^{\mathrm{a}, \mathrm{d}}$, Umberto diPorzio ${ }^{\mathrm{b}}$, Carla Perrone-Capano ${ }^{\mathrm{b}, \mathrm{c}}$, Giovanni Laviola ${ }^{\mathrm{a}}$, \\ Jean-Luc Dreyer ${ }^{\mathrm{d}}$, Walter Adriani ${ }^{\mathrm{a}, *}$ \\ a Dept. Cell Biology E Neurosciences, Istituto Superiore di Sanità, Rome, Italy \\ ${ }^{\mathrm{b}}$ Institute of Genetics and Biophysics, CNR, Naples, Italy \\ ${ }^{c}$ Dept. Biological Sciences, University “Federico II", Naples, Italy

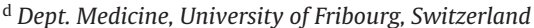 \\ e Bambino Gesù Children's Hospital IRCCS, Rome, Italy
}

\section{H I G H L I G H T S}

- Lenti-SERT vectors were designed to suppress SERT gene expression in vivo.

- We evaluated a rat model of ADHD through hippocampal inoculation of Lenti-SERT.

- Lenti-SERT rats exhibited less pronounced peaks of circadian activity than controls.

- Lenti-SERT rats displayed a transient decrease in cognitive impulsivity.

- Such phenotype is consistent both with 5-HT manipulations and hippocampal lesions.

Brain serotonin (5-HT) systems modulate emotional, motivational and cognitive processes. Mutations in the serotonin transporter (SERT) gene have been associated with susceptibility towards the development of several psychiatric disorders, both in humans and animal models. Present approach exploited a bilateral intra-hippocampus stereotaxic inoculation of lentiviruses, for enduring in vivo silencing of SERT. Control rats were bilaterally inoculated with heat-inactivated lentiviruses. These Lenti-SERT vectors were intended to eventually manipulate the neurotransmitter reuptake at synaptic level, thus enhancing tonic 5-HT transmission. We investigated whether such manipulation could induce behavioural alterations relevant to the modelling of ADHD, in particular symptoms of hyperactivity and impulsivity. Wistar rats were monitored for spontaneous home-cage locomotor activity and studied for impulsivity (Intoleranceto-Delay task). Results show that rats inoculated with Lenti-SERT vectors exhibited less pronounced circadian peaks of activity than controls. Moreover, Lenti-SERT compared to control rats exhibited a transient increase in choice for a delayed-larger reward over an immediate-small reward. This suggests that enhanced hippocampal serotonergic transmission produced a profile of restfulness and a decrease in cognitive impulsivity. This phenotype is consistent with available data both on 5-HT manipulations and hippocampal lesions. In conclusion, present findings may possibly disclose novel avenues towards the development of innovative therapeutical approaches for behavioural symptoms relevant to ADHD.

* Corresponding author at: viale Regina Elena 299, I-00161 Rome, Italy. Tel.: +39 0649903171

E-mail address: walter.adriani@iss.it (W. Adriani).

\section{Introduction}

Attention-deficit/hyperactivity disorder (ADHD) is a heterogeneous syndrome, affecting $2-5 \%$ of infants and adolescents as well as about $2 \%$ of adults worldwide [59]. Together with inattention and motor hyperactivity, one key symptom is impulsivity, a multifaceted concept implicated in many disorders characterized by inappropriate inhibitory control [16]. It has been suggested that 
impulsivity is not a unitary construct, but describes a range of behaviours and encompasses a variety of related phenomena that may differ in their biological basis [12,60].

The serotonergic system is well known for modulation of emotional, cognitive and motivational processes [11]. Dysfunctions in this system play a crucial role in many psychiatric disorders, including affective and impulse-control disorders [39,50]. By mediating the interplay between limbic and cognitive loops, forebrain serotonin (5-HT) has a key role in the top-down inhibitory control over behavioural initiation and execution, which is important for withholding of instinctive reactions and for an appropriate feedback regulation of behaviour $[11,22]$. As such, it is central to psychomotor control over subcortical processing of reward and reinforcement [47].

Permanent in vivo interference with gene and protein expression and function is nowadays possible using lentiviral vectors [42]. We have recently used this approach exploiting brain inoculation of lentiviruses targeting the dopamine transporter (DAT): its overexpression in rats' nucleus accumbens leads to an impulsive and risk-prone phenotype $[1,2]$. Similarly, we prepared a novel vector designed to suppress the serotonin transporter (SERT) gene expression. Indeed, mutations in the SERT gene influence the rate of 5-HT reuptake and have been associated with susceptibility towards the development of several psychiatric disorders [10,53]. Specifically, a common polymorphism (5-HTTLPR), localised in the promoter of the SERT-encoding gene, gives rise to two major allelic variants [31] that significantly change the probability of being affected by ADHD $[18,40,54]$. In particular, the hippocampus was selected as the inoculation site, since its lesions are known to cause hyperactivity and impulsive choice in rats (e.g. $[9,27,46])$.

Experimental studies with different serotonergic manipulations have demonstrated an inverse relationship between 5-HT levels and impulsivity, with a reduction in the neurotransmitter causing an increase in impulsivity and vice versa (e.g. $[8,55])$. However, in humans, an increase in impulsivity appears to be associated with the short (s) allelic variant of 5-HTTLPR, leading to reduced SERT gene transcription $[41,51,57]$. Thus, we aimed to determine whether a partial silencing of the SERT-encoding gene within the hippocampus could induce alterations relevant to the modelling of ADHD, in particular symptoms of hyperactivity and impulsivity. Specifically, for measuring behavioural impulsivity, the Intolerance-to-Delay (ID) task was used, in which impulsive subjects are detected by their intolerance to periods of forced waiting before the delivery of a large reward [17]. Indeed, delay-discounting paradigms in general are among the most successfully utilised tools for measurement of impulsive choice [60].

\section{Materials and methods}

\subsection{Lenti-SERT vectors}

\subsubsection{Construction of pTK431-SERT-siRNAs}

To silence SERT expression in vivo, three targets were designed according to the SERT mRNA sequence. The following targets were selected, based on Hannon's design criterion: 1st target: bp64-29, 2nd target: bp2667-2629, 3rd target: bp1805-1829. To each oligo, a XhoI restriction site was added at $3^{\prime}$ and a U6-3'-specific 10 mer at $5^{\prime}$. Using the pSilencer 1.0-U6 (Ambion, UK) as a template and a U6 promoter-specific forward primer containing BamHI restriction site (5'-CGC CGC CGC GGA TCC CGC TCT AGA ACT AGT GC-3'), each siRNA target was added to the mouse U6 promoter by PCR, using the following program: $120 \mathrm{~s}$ at $94{ }^{\circ} \mathrm{C}$ (initial denaturation) followed by 35 cycles ( $45 \mathrm{~s}$ at $94^{\circ} \mathrm{C}, 45 \mathrm{~s}$ at $64^{\circ} \mathrm{C}$ and $45 \mathrm{~s}$ at $72^{\circ} \mathrm{C}$ ) in $4 \%$ dimethyl sulfoxide (Sigma, Switzerland). The PCR product was digested with BamHI and XhoI, cloned into similar sites into pTK431, and sequenced to verify the integrity of each construct.

\subsubsection{Lentivirus production}

The vector plasmids (pTK431-U6-siSERT1, pTK431-U6-siSERT2, pTK431-U6-siSERT3 and pTK433-GFP), together with the packaging construct plasmid p891 and the envelope plasmid PMD2-G, were co-transfected into HEK293T cells to produce the viral particles [6,7]. Once harvested and concentrated, these viral particles were tested in vitro. The experiment of infection and transfection was run with 3,10 and $25 \mu \mathrm{l}$ of each lentivirus stock (LV-siSERT1, LV-siSERT2, LV-siSERT3) and $20 \mu \mathrm{l}$ of a mix of the three siLVs. HEK293T cells were plated and infected with LV-siSERTs and then transfected with a plasmid that expresses SERT. The siLVs were added with Polybrene (Sigma, Switzerland, at $10 \mu \mathrm{g} / \mathrm{ml}$ final concentration) to the cells. After $7 \mathrm{~h}$, cells were transfected with pcDNA3-rat-SERT ( $2 \mu \mathrm{g} /$ well). The next day, cells were subjected to total RNA isolation, reverse transcription and real-time PCR, to quantify SERT expression/silencing (see Supplementary Data).

\subsection{Subjects}

Seventeen adult male Wistar rats ( $400 \mathrm{~g}$; for housing conditions, see Supplementary Data) were randomly assigned to experimental groups: one group received bilateral inoculation of Lenti-SERT vectors ( $1 \mu \mathrm{l}$ of a mix of the three LV-siSERTs) intended to abolish the genetic expression of SERT. Inoculations were made bilaterally at coordinates $\mathrm{AP}-3.3, \mathrm{ML} \pm 2.2, \mathrm{DV}-4.0$ from bregma [45]. The other group (controls) received a bilateral inoculation of heat-inactivated lentiviruses $(1 \mu \mathrm{l})$ at the same coordinates (see Supplementary Data). After surgery, rats were single-housed and left undisturbed for at least one month prior to behavioural experiments.

\subsection{Circadian cycle}

Rats were continuously monitored for spontaneous home-cage locomotor activity $[3,4]$ by means of an automatic device equipped with small passive infrared sensors placed on a standard rack over the top of each home-cage (ActiviScope system; TechnoSmart, Rome, Italy). These sensors $(20 \mathrm{~Hz})$ detected any movement of rats: scores were automatically divided into 60-min intervals. The spontaneous home-cage activity was continuously measured in Lenti-SERT and control subjects, starting from day 50 after inoculation and over 15 days. From this period, we extracted the central 5-days interval, from which a mean day was calculated.

\subsection{ID task for impulsivity}

Two months later, rats were daily tested (between 10:30 and 15:30) exploiting classical Skinner-boxes (for apparatus and foodrestriction schedule, see Supplementary Data). Nose-poking in one hole (termed "Small \& Soon", SS) resulted in the immediate delivery of one pellet in one magazine, whereas nose-poking in the other hole (termed "Large \& Late", LL) resulted in the delivery of five pellets in the other magazine. After nose-poking and before food delivery, the chamber light corresponding to the nose-poked hole was switched on for $1 \mathrm{~s}$. Following food delivery, the corresponding magazine light was turned on for $30 \mathrm{~s}$, to signal the length of timeout (TO) during which additional nose-poking was recorded but had no scheduled consequences (i.e. inadequate, see Supplementary Data) $[49,50]$. The testing phase was preceded by three training sessions at delay $0 \mathrm{~s}$, which allowed subjects to reach a significant preference for LL. During the testing phase (eight daily sessions, 40 min each), a delay was inserted between nose-poking in the LL hole and delivery of the 5-pellet reward. The chamber light over the 
LL hole was kept on to signal the entire length of this delay, which was fixed for each daily session and was progressively increased across subsequent days, from $0 \mathrm{~s}$ to $90 \mathrm{~s}$. The small-reward delivery was unchanged.

\subsection{Data analysis}

Data were analyzed using repeated-measures ANOVA. The general model was: 24-level time (hour; circadian cycle study) or 8 -level session (delay; ID task) $\times 2$-level group (treatment). Statistical analysis was performed using Statview II (Abacus Concepts, USA). Data are expressed as mean \pm SEM. Level of significance was set at $P<0.05$. Multiple post hoc comparisons were performed with the Tukey's HSD test.

\section{Results}

\subsection{Quantification of SERT silencing}

Compared to SERT expression level in transfected HEK293T cells $(100 \%)$, infection with the three siLVs generated the following averaged mRNA expression levels: 78\% for LV-siSERT1, 64\% for LV-siSERT2, 91\% for LV-siSERT3 and 78\% for their mix.

\subsection{Circadian cycle}

Data collected from the automated ActiviScope system showed that circadian activity patterns differed depending on treatment (Fig. 1A). Lenti-SERT animals were significantly less active than controls at specific time intervals throughout the day. Specifically, the ANOVA yielded significance for group, time and their interaction (treatment: $F_{(1,15)}=9.11, P=0.0087$; time: $F_{(23,345)}=58.81$, $P<0.0001$; time $\times$ treatment: $\left.F_{(23,345)}=2.46, P=0.0003\right)$. Lenti-SERT animals showed no differences from controls in the expected diurnal rest pattern, as well as in the well-known peak observed at light switch off. As for nocturnal activity pattern, the progressive increase towards the major level of activity (between 10:00 and 15:00) was significantly less marked in the LentiSERT when compared to the control group. A second peak was observed in both groups between 17:00 and 20:00, and again the Lenti-SERT rats were significantly less active than controls.

\subsection{ID task for impulsivity: preference (\%) for LL reward}

As expected, all animals showed a clear-cut shift in preference towards the SS reward as the delay length increased (group: $F_{(1,14)}=1.52, P=0.2372$; session: $F_{(7,98)}=52.43, P<0.0001$; session $\times$ group: $\left.F_{(7,98)}=0.57, P=0.7745\right)$. However, animals belonging to the Lenti-SERT group seemed to display such a shift later (at delay of $45 \mathrm{~s}$ ) than did control animals (at delay of $30 \mathrm{~s}$ ). Post hoc comparisons, drawn at this latter value of delay, revealed that LL hole was still significantly preferred by Lenti-SERT rats, whereas a delay-induced shift was already pronounced in the corresponding controls (Fig. 1B). Therefore, we may conclude that Lenti-SERT subjects showed a transiently less impulsive choice than controls.

\section{Discussion}

Presently, a new approach exploited a lentivirus-mediated silencing of SERT within the hippocampus (HPC), with the aim of generating an animal model for impulsivity and hyperactivity, which in turn are symptoms relevant to ADHD. Such model is to some extent similar to a local knockout of the SERT gene [23], with
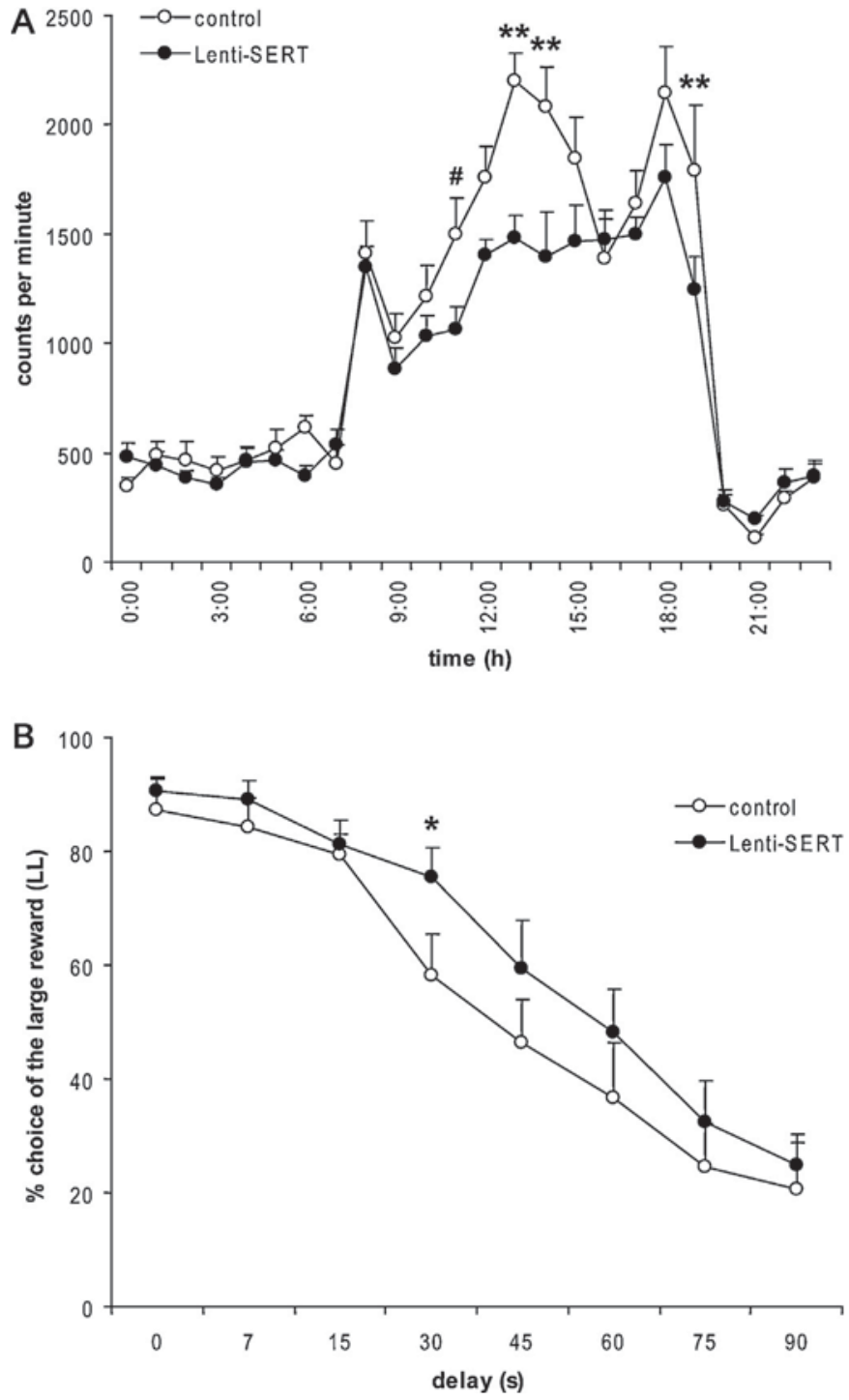

Fig. 1. (A) Circadian activity cycle. Circadian activity (mean \pm SEM) in Lenti-SERT $(n=11)$ and control $(n=6)$ rats, left undisturbed inside their home-cages (counts per minute, summed up to 1 -h points). The 24 -h profile was obtained by averaging five days of continuous registration. Marked differences were found in the nocturnal activity pattern: activity levels were significantly lower in Lenti-SERT rats compared to controls. ${ }^{* *} P<0.01 ;{ }^{*} 0.05<P<0.1$ in post hoc test. (B) Impulsivity in the ID task. Mean $( \pm$ SEM) choice $(\%)$ of the large reward (LL), as a function of absolute delay duration $(s)$, shown by Lenti-SERT $(n=10)$ and control $(n=6)$ animals. Lenti-SERT rats experienced a clear-cut intolerance at a longer delay than did the corresponding controls. ${ }^{*} P<0.05$ in post hoc test.

the advantages that silencing is performed in the HPC of already adult animals; therefore, this approach may imply less compensatory mechanisms compared to breeding knockout models. We emphasize that our aim was to induce a partial (not a total) silencing of the SERT-encoding gene, thus mimicking to some extent the short (s) allelic variant of 5-HTTLPR. Our results show that rats inoculated with Lenti-SERT exhibited decreased spontaneous locomotor activity and decreased cognitive impulsivity.

SERT, which selectively removes 5 -HT out of the synaptic cleft, is a major determinant of serotonergic signalling efficiency, related to the neurotransmitter concentration (e.g. [52]). Studies in vitro showed that the s-variant of the 5-HTTLPR was associated with reduced SERT gene transcription efficiency, resulting in reduced SERT levels and reduced 5-HT uptake. Consequently, the extracellular levels of 5-HT were higher compared to the l-variant (e.g. $[20,31])$. In the present study, the silencing of SERT gene in the 
Lenti-SERT rats was intended to produce an improvement of serotonergic tone, which we hypothesized to mimic, to a certain extent, the condition of human subjects carrying the s-variant. Similarly, homozygous SERT ${ }^{-1}$ rodents, which are widely considered to resemble the effects of the s/s genotype in humans [25], show alterations in multiple neurobehavioral domains, including behavioural inhibition and decision making [28].

\subsection{Circadian rhythm of activity}

By analyzing the circadian pattern, the hypolocomotor effect in Lenti-SERT rats appears to be "intrinsic" to animals' physiology, since it was observed in home-cage conditions and it was stable across days. It was specifically localized around two out of the three daily peaks of activity (in the middle of the dark phase and before the start of the lit period).

5-HT is known to have complex actions with respect to control of activity levels [36], although not all forms of locomotion are equally dependent on central 5-HT transmission [14]. For the purposes of the present paper, it is worthwhile to report that SERT ${ }^{-1-}$ mice show decreased home-cage activity [21]. Increases in homecage activity have been reported following depletion of brain 5-HT. For example, both PCPA (parachlorophenylalanine, an inhibitor of the 5-HT-synthesizing enzyme) administration and lesions of the median raphe induce an enhancement of locomotor activity in familiar environments $[15,19,26]$. Conversely, chronic treatment with selective serotonin reuptake inhibitors (SSRIs; that, by inhibiting the reuptake, increase 5-HT extracellular level) caused rats to run significantly less on a running-wheel in their home-cages [5]. We conclude that decreased spontaneous activity exhibited by Lenti-SERT rats is consistent with available data on 5-HT manipulations in rodents. Moreover, HPC lesions are known to increase (nocturnal) home-cage activity (e.g. [27]). Thus, the decrease in home-cage activity observed in present Lenti-SERT rats is also consistent with a possibly improved HPC function, due in turn to higher amount of extracellular 5-HT in this brain area.

\subsection{Cognitive impulsivity}

Lenti-SERT rats preferred a delayed-larger reward over an immediate-small reward when the waiting interval was $30 \mathrm{~s}$, suggesting that an enhanced HPC serotonergic transmission rendered these animals slightly less impulsive. Central 5-HT manipulations are reported to affect the ability to wait for reinforcement in studies performed with paradigms for either impulsive-choice (e.g. [8,38]) or impulsive-action (e.g. $[13,15])$. For example, lesions of 5-HT pathways $[8,15,38]$ as well as PCPA administration $[8,13]$ resulted in increased impulsivity. Moreover, SERT $^{-1-}$ rats showed increased correct response latency and decreased premature responding in the 5-choice serial reaction time task (5-CSRTT; [24]). Based on available data, it has been speculated that SERT ${ }^{-1-}$ rats would also show improved "waiting" when reward is delayed [28]. Consistently, acute increase in 5-HT levels, through SSRIs treatment, resulted in decreased impulsivity in a reward-delay task [8].

Impulsive behaviour has been associated not only with orbitofrontal cortex (OFC) lesions (e.g. $[29,37])$ but also with HPC lesions (e.g. [9,46]). McHugh and colleagues [35] have shown, using a spatial task involving a choice between a delayed-larger vs. an immediate-small rewards, that both OFC- or HPC-lesioned rats showed an increase in impulsive choice. Interestingly, the same group [34] recently reported that, using a non-spatial version of the same task, HPC-lesioned rats exhibited impulsive choice whilst OFC-lesioned rats did not. Thus, the role of OFC in choice with delayed rewards could be more limited [61], and that of the HPC more extensive, than classically thought; this is probably related to HPC role in temporal information processing [34,61]. We may conclude that the transient increase in choice for a delayed reward, exhibited by Lenti-SERT, supports the hypothesis of an inverse relationship between 5-HT and impulsivity [55], being consistent with available data both on 5-HT manipulations (including pathway lesions, SSRI administration and SERT knockout) and HPC lesions.

\subsection{Clinical implications}

Studies on the association between impulsivity and the polymorphic variants of 5HTTLPR in humans found rather controversial results [30]: many studies describe a significant association between impulsivity and the s-allele of 5-HTTLPR $[41,51,57]$ but about as many studies do not find such a relationship $[44,48,58]$. As a whole, it may be not surprising that the s-allele could be associated with more impulsive performance in clinical research, given the anatomical complexity of the serotonergic projections, the variety of receptor families and subtypes, and the consequential breadth of its functions.

Moreover, although 5-HTTLPR studies in human lymphoblasts and platelets have shown that the s-variant is associated with reduced SERT expression and function (e.g. [20,31]), no definitive data exist showing that the s/s genotype is associated in vivo with decreased SERT expression in human CNS. Indeed, post-mortem studies on brain SERT mRNA levels (e.g. [32,33]) and PET studies on central SERT binding (e.g. $[43,56]$ ) have not always confirmed that the 5-HTTLPR variants have different effects on SERT transcription.

\section{Conclusions}

In summary, by allowing a selective SERT silencing in rats' hippocampus, the present approach will hopefully contribute to a further understanding of the biological mechanisms underlying psychiatric disorders. The same approach is worth to be explored for modelling other pathologies, above all mood disorders (e.g. depression, anxiety), which are also related to an alteration of SERT function. Noteworthy, rats transfected with a SERT silencer are more restful in home-cage conditions and display more "patience" when facing a reward delay. This may possibly disclose novel avenues towards the development of innovative therapeutical approaches for behavioural symptoms relevant to ADHD.

\section{Acknowledgements}

Funding: Under-40 "ADHD-sythe" and ERA-net "NeuroGenMRI" (to WA); FIRB-RBIN062YH4 and PRIN-2009TBCZJB_003 (to UdP).

\section{Appendix A. Supplementary data}

Supplementary data associated with this article can be found, in the online version,

\section{References}

[1] W. Adriani, F. Boyer, L. Gioiosa, S. Macri, J.L. Dreyer, G. Laviola, Increased impulsive behavior and risk proneness following lentivirus-mediated dopamine transporter over-expression in rats' nucleus accumbens, Neuroscience 159 (2009) 47-58.

[2] W. Adriani, F. Boyer, D. Leo, R. Canese, F. Podo, C. Perrone-Capano, J.L. Dreyer, G. Laviola, Social withdrawal and gambling-like profile after lentiviral manipulation of DAT expression in the rat accumbens, Int. J. Neuropsychopharmacol. 13 (2010) 1329-1342.

[3] W. Adriani, A. Caprioli, O. Granstrem, M. Carli, G. Laviola, The spontaneously hypertensive-rat as an animal model of ADHD: evidence for impulsive and non-impulsive subpopulations, Neurosci. Biobehav. Rev. 27 (2003) 639-651.

[4] W. Adriani, D. Travaglini, E. Lacivita, L. Saso, M. Leopoldo, G. Laviola, Modulatory effects of two novel agonists for serotonin receptor 7 on emotion, motivation and circadian rhythm profiles in mice, Neuropharmacology 62 (2012) 833-842. 
[5] M. Altemus, J.R. Glowa, E. Galliven, Y.M. Leong, D.L. Murphy, Effects of serotonergic agents on food-restriction-induced hyperactivity, Pharmacol. Biochem. Behav. 53 (1996) 123-131.

[6] A. Bahi, F. Boyer, C. Gumy, T. Kafri, J.L. Dreyer, In-vivo gene delivery of urokinase-type plasminogen activator with regulatable lentivirus induces behavioural changes in chronic cocaine administration, Eur. J. Neurosci. 20 (2004) 3473-3488.

[7] A. Bahi, F. Boyer, T. Kafri, J.L. Dreyer, CD81-induced behavioural changes during chronic cocaine administration: in-vivo gene delivery with regulatable lentivirus, Eur. J. Neurosci. 19 (2004) 1621-1633.

[8] J. Bizot, C. Le Bihan, A.J. Puech, M. Hamon, M. Thiebot, Serotonin and tolerance to delay of reward in rats, Psychopharmacology 146 (1999) 400-412.

[9] T.H. Cheung, R.N. Cardinal, Hippocampal lesions facilitate instrumental learning with delayed reinforcement but induce impulsive choice in rats, BMC Neurosci. 6 (2005) 36

[10] D.A. Collier, G. Stober, T. Li, A. Heils, M. Catalano, D. Di Bella, M.J. Arranz, R.M Murray, H.P. Vallada, D. Bengel, C.R. Muller, G.W. Roberts, E. Smeraldi, G. Kirov, P. Sham, K.P. Lesch, A novel functional polymorphism within the promoter of the serotonin transporter gene: possible role in susceptibility to affective disorders, Mol. Psychiatr. 1 (1996) 453-460.

[11] R. Cools, K. Nakamura, N.D. Daw, Serotonin and dopamine: unifying affective, activational, and decision functions, Neuropsychopharmacology 36 (2011) 98-113.

[12] J.W. Dalley, A.C. Mar, D. Economidou, T.W. Robbins, Neurobehavioral mechanisms of impulsivity: fronto-striatal systems and functional neurochemistry, Pharmacol. Biochem. Behav. 90 (2008) 250-260.

[13] F. Denk, M.E. Walton, K.A. Jennings, T. Sharp, M.F. Rushworth, D.M. Bannerman, Differential involvement of serotonin and dopamine systems in cost-benefit decisions about delay or effort, Psychopharmacology 179 (2005) 587-596.

[14] H.C. Dringenberg, E.L. Hargreaves, G.B. Baker, R.K. Cooley, C.H. Vanderwolf, pChlorophenylalanine-induced serotonin depletion: reduction in exploratory locomotion but no obvious sensory-motor deficits, Behav. Brain Res. 68 (1995) 229-237.

[15] D.M. Eagle, O. Lehmann, D.E. Theobald, Y. Pena, R. Zakaria, R. Ghosh, J.W. Dalley, T.W. Robbins, Serotonin depletion impairs waiting but not stop-signal reaction time in rats: implications for theories of the role of 5-HT in behavioral inhibition, Neuropsychopharmacology 34 (2009) 1311-1321.

[16] J.L. Evenden, Varieties of impulsivity, Psychopharmacology 146 (1999) 348-361.

[17] J.L. Evenden, C.N. Ryan, The pharmacology of impulsive behaviour in rats: the effects of drugs on response choice with varying delays of reinforcement, Psychopharmacology 128 (1996) 161-170.

18] S.V. Faraone, S.A. Khan, Candidate gene studies of ADHD, attentiondeficit/hyperactivity disorder, J. Clin. Psychiatr. 67 (Suppl. 8) (2006) 13-20.

[19] H.C. Fibiger, B.A. Campbell, The effect of para-chlorophenylalanine on spontaneous locomotor activity in the rat, Neuropharmacology 10 (1971) 25-32.

[20] B.D. Greenberg, T.J. Tolliver, S.J. Huang, Q. Li, D. Bengel, D.L. Murphy, Genetic variation in the serotonin transporter promoter region affects serotonin uptake in human blood platelets, Am. J. Med. Gen. 88 (1999) 83-87.

[21] A.R. Hariri, A. Holmes, Genetics of emotional regulation: the role of the serotonin transporter in neural function, Trends Cog. Sci. 10 (2006) 182-191.

[22] J.R. Homberg, Serotonin and decision making processes, Neurosci. Biobehav. Rev. 36 (2012) 218-236.

[23] J.R. Homberg, J.D. Olivier, B.M. Smits, J.D. Mul, J. Mudde, M. Verheul, O.F Nieuwenhuizen, A.R. Cools, E. Ronken, T. Cremers, A.N. Schoffelmeer, B.A. Ellenbroek, E. Cuppen, Characterization of the serotonin transporter knockout rat: a selective change in the functioning of the serotonergic system, Neuroscience 146 (2007) 1662-1676.

[24] J.R. Homberg, T. Pattij, M.C. Janssen, E. Ronken, S.F. De Boer, A.N. Schoffelmeer, E. Cuppen, Serotonin transporter deficiency in rats improves inhibitory control but not behavioural flexibility, Eur. J. Neurosci. 26 (2007) 2066-2073.

[25] J.R. Homberg, R. van den Bos, E. den Heijer, R. Suer, E. Cuppen, Serotonin transporter dosage modulates long-term decision-making in rat and human, Neuropharmacology 55 (2008) 80-84.

[26] B.L. Jacobs, C.A. Fornal, Serotonin and motor activity, Curr. Opin. Neurobiol. 7 (1997) 820-825.

[27] H. Jeltsch, F. Bertrand, C. Lazarus, J.C. Cassel, Cognitive performances and locomotor activity following dentate granule cell damage in rats: role of lesion extent and type of memory tested, Neurobiol. Learn. Mem. 76 (2001) 81-105.

[28] A.V. Kalueff, J.D. Olivier, L.J. Nonkes, J.R. Homberg, Conserved role for the serotonin transporter gene in rat and mouse neurobehavioral endophenotypes, Neurosci. Biobehav. Rev. 34 (2010) 373-386.

[29] S. Kheramin, S. Body, S. Mobini, M.Y. Ho, D.N. Velazquez-Martinez, C.M. Bradshaw, E. Szabadi, J.F. Deakin, I.M. Anderson, Effects of quinolinic acid-induced lesions of the orbital prefrontal cortex on inter-temporal choice: a quantitative analysis, Psychopharmacology 165 (2002) 9-17.

[30] G.M. Lage, L.F. Malloy-Diniz, L.O. Matos, M.A. Bastos, S.S. Abrantes, H. Correa, Impulsivity and the 5-HTTLPR polymorphism in a non-clinical sample, PloS One 6 (2011) e16927.

[31] K.P. Lesch, D. Bengel, A. Heils, S.Z. Sabol, B.D. Greenberg, S. Petri, J. Benjamin, C.R. Muller, D.H. Hamer, D.L. Murphy, Association of anxiety-related traits with a polymorphism in the serotonin transporter gene regulatory region, Science 274 (1996) 1527-1531.

[32] J.E. Lim, A. Papp, J. Pinsonneault, W. Sadee, D. Saffen, Allelic expression of serotonin transporter (SERT) mRNA in human pons: lack of correlation with the polymorphism SERTLPR, Mol. Psychiatr. 11 (2006) 649-662.
[33] K.Y. Little, D.P. McLaughlin, L. Zhang, C.S. Livermore, G.W. Dalack, P.R. McFinton, Z.S. DelProposto, E. Hill, B.J. Cassin, S.J. Watson, E.H. Cook, Cocaine, ethanol, and genotype effects on human midbrain serotonin transporter binding sites and mRNA levels, Am. J. Psychiatr. 155 (1998) 207-213.

[34] T.Y. Mariano, D.M. Bannerman, S.B. McHugh, T.J. Preston, P.H. Rudebeck, S.R. Rudebeck, J.N. Rawlins, M.E. Walton, M.F. Rushworth, M.G. Baxter, T.G. Campbell, Impulsive choice in hippocampal but not orbitofrontal cortex-lesioned rats on a nonspatial decision-making maze task, Eur. J. Neurosci. 30 (2009) $472-484$.

[35] S.B. McHugh, T.G. Campbell, A.M. Taylor, J.N. Rawlins, D.M. Bannerman, A role for dorsal and ventral hippocampus in inter-temporal choice cost-benefit decision making, Behav. Neurosci. 122 (2008) 1-8.

[36] L. Mignon, W.A. Wolf, Postsynaptic 5-HT(1A) receptors mediate an increase in locomotor activity in the monoamine-depleted rat, Psychopharmacology 163 (2002) 85-94.

[37] S. Mobini, S. Body, M.Y. Ho, C.M. Bradshaw, E. Szabadi, J.F. Deakin, I.M. Anderson, Effects of lesions of the orbitofrontal cortex on sensitivity to delayed and probabilistic reinforcement, Psychopharmacology 160 (2002) 290-298.

[38] S. Mobini, T.J. Chiang, A.S. Al-Ruwaitea, M.Y. Ho, C.M. Bradshaw, E. Szabadi, Effect of central 5-hydroxytryptamine depletion on inter-temporal choice: a quantitative analysis, Psychopharmacology 149 (2000) 313-318.

[39] R.D. Oades, Frontal, temporal and lateralized brain function in children with attention-deficit hyperactivity disorder: a psychophysiological and neuropsychological viewpoint on development, Behav. Brain Res. 94 (1998) 83-95.

[40] R.D. Oades, J. Lasky-Su, H. Christiansen, S.V. Faraone, E.J. Sonuga-Barke, T. Banaschewski, W. Chen, R.J. Anney, J.K. Buitelaar, R.P. Ebstein, B. Franke, M. Gill, A. Miranda, H. Roeyers, A. Rothenberger, J.A. Sergeant, H.C. Steinhausen, E.A. Taylor, M. Thompson, P. Asherson, The influence of serotonin- and other genes on impulsive behavioral aggression and cognitive impulsivity in children with attention-deficit/hyperactivity disorder (ADHD): findings from a family-based association test (FBAT) analysis, Behav. Brain Funct. 4 (2008) 48.

[41] M. Paaver, N. Nordquist, J. Parik, M. Harro, L. Oreland, J. Harro, Platelet MAO activity and the 5-HTT gene promoter polymorphism are associated with impulsivity and cognitive style in visual information processing, Psychopharmacology 194 (2007) 545-554.

[42] F. Park, Lentiviral vectors: are they the future of animal transgenesis? Physiol. Genomics 31 (2007) 159-173.

[43] R.V. Parsey, R.S. Hastings, M.A. Oquendo, X. Hu, D. Goldman, Y.Y. Huang, N Simpson, J. Arcement, Y. Huang, R.T. Ogden, R.L. Van Heertum, V. Arango, J.J. Mann, Effect of a triallelic functional polymorphism of the serotonintransporter-linked promoter region on expression of serotonin transporter in the human brain, Am. J. Psychiatr. 163 (2006) 48-51.

[44] A.A. Patkar, W.H. Berrettini, M. Hoehe, C.C. Thornton, E. Gottheil, K. Hill, S.P. Weinstein, Serotonin transporter polymorphisms and measures of impulsivity, aggression, and sensation seeking among AfricanAmerican cocaine-dependent individuals, Psychiatr. Res. 110 (2002) 103-115.

[45] G. Paxinos, C. Watson, The Rat Brain in Stereotaxic Coordinates, Academic Press, San Diego, 1998.

[46] J.N. Rawlins, J. Feldon, S. Butt, The effects of delaying reward on choice preference in rats with hippocampal or selective septal lesions, Behav. Brain Res. 15 (1985) 191-203.

[47] K.R. Ridderinkhof, W.P.M. van den Wildenberg, S.J. Segalowitz, C.S. Carter, Neurocognitive mechanisms of cognitive control: the role of prefrontal cortex in action selection, response inhibition, performance monitoring, and rewardbased learning, Brain Cogn. 56 (2004) 129-140.

[48] J.P. Roiser, U. Muller, L. Clark, B.J. Sahakian, The effects of acute tryptophan depletion and serotonin transporter polymorphism on emotional processing in memory and attention, Int. J. Neuropsychopharmacol. 10 (2007) 449-461.

[49] T. Sagvolden, Behavioral validation of the spontaneously hypertensive rat (SHR) as an animal model of attention-deficit/hyperactivity disorder (AD/HD), Neurosci. Biobehav. Rev. 24 (2000) 31-39.

[50] T. Sagvolden, J.A. Sergeant, Attention deficit/hyperactivity disorder-from brain dysfunctions to behaviour, Behav. Brain Res. 94 (1998) 1-10.

[51] K. Sakado, M. Sakado, T. Muratake, C. Mundt, T. Someya, A psychometrically derived impulsive trait related to a polymorphism in the serotonin transporter gene-linked polymorphic region (5-HTTLPR) in a Japanese nonclinical population: assessment by the Barratt impulsiveness scale (BIS), Am. J. Med. Genet. B Neuropsychiatr. Genet. 121B (2003) 71-75.

[52] P. Schloss, D.C. Williams, The serotonin transporter: a primary target for antidepressant drugs, J. Psychopharmacol 12 (1998) 115-121.

[53] G. Seeger, P. Schloss, M.H. Schmidt, Functional polymorphism within the promotor of the serotonin transporter gene is associated with severe hyperkinetic disorders, Mol. Psychiatr. 6 (2001) 235-238.

[54] E.J. Sonuga-Barke, R. Kumsta, W. Schlotz, J. Lasky-Su, R. Marco, A. Miranda, F. Mulas, R.D. Oades, T. Banaschewski, U. Mueller, P. Andreou, H. Christiansen, I. Gabriels, H. Uebel, J. Kuntsi, B. Franke, J. Buitelaar, R. Ebstein, M. Gill, R. Anney, H. Roeyers, A. Rothenberger, J. Sergeant, H.C. Steinhausen, P. Asherson, S.V. Faraone, A functional variant of the serotonin transporter gene (SLC6A4) moderates impulsive choice in attention-deficit/hyperactivity disorder boys and siblings, Biol. Psychiatr. 70 (2011) 230-236.

[55] P. Soubrié, Reconciling the role of central serotonin neurons in human and animal behavior, Behav. Brain Sci. 9 (1986) 319-364.

[56] C.H. van Dyck, R.T. Malison, J.K. Staley, L.K. Jacobsen, J.P. Seibyl, M. Laruelle, R.M. Baldwin, R.B. Innis, J. Gelernter, Central serotonin transporter availability 
measured with [123I]beta-CIT SPECT in relation to serotonin transporter genotype, Am. J. Psychiatr. 161 (2004) 525-531.

[57] E. Walderhaug, A.I. Herman, A. Magnusson, M.J. Morgan, N.I. Landro, The short allele of the serotonin transporter polymorphism and acute tryptophan depletion both increase impulsivity in men, Neurosci. Lett. 473 (2010) 208-211.

[58] E. Walderhaug, A. Magnusson, A. Neumeister, J. Lappalainen, H. Lunde, H. Refsum, N.I. Landro, Interactive effects of sex and 5-HTTLPR on mood and impulsivity during tryptophan depletion in healthy people, Biol. Psychiatr. 62 (2007) 593-599.
[59] L. Wichstrom, T.S. Berg-Nielsen, A. Angold, H.L. Egger, E. Solheim, T.H. Sveen, Prevalence of psychiatric disorders in preschoolers, J. Child Psychol. Psychiatr 53 (2012) 695-705.

[60] C.A. Winstanley, D.M. Eagle, T.W. Robbins, Behavioral models of impulsivity in relation to ADHD: translation between clinical and preclinical studies, Clin. Psychol. Rev. 26 (2006) 379-395.

[61] C.A. Winstanley, D.E. Theobald, R.N. Cardinal, T.W. Robbins, Contrasting roles of basolateral amygdala and orbitofrontal cortex in impulsive choice, J. Neurosci. 24 (2004) 4718-4722. 